\title{
A FAMILY OF $q$-SUPERCONGRUENCES MODULO THE CUBE OF A CYCLOTOMIC POLYNOMIAL
}

\author{
VICTOR J. W. GUO ${ }^{\circledR}$ and MICHAEL J. SCHLOSSER ${ }^{\circledR}$
}

(Received 6 June 2021; accepted 14 July 2021; first published online 3 September 2021)

\begin{abstract}
We establish a family of $q$-supercongruences modulo the cube of a cyclotomic polynomial for truncated basic hypergeometric series. This confirms a weaker form of a conjecture of the present authors. Our proof employs a very-well-poised Karlsson-Minton type summation due to Gasper, together with the "creative microscoping' method introduced by the first author in recent joint work with Zudilin.
\end{abstract}

2020 Mathematics subject classification: primary 33D15; secondary 11A07, 11B65.

Keywords and phrases: basic hypergeometric series, supercongruences, $q$-congruences, cyclotomic polynomial, Gasper's summation.

\section{Introduction}

In 1914, Ramanujan [11] mysteriously stated some representations of $1 / \pi$, such as

$$
\sum_{k=0}^{\infty}(6 k+1) \frac{\left(\frac{1}{2}\right)_{k}^{3}}{k !^{3} 4^{k}}=\frac{4}{\pi}
$$

where $(a)_{n}=a(a+1) \cdots(a+n-1)$ denotes the rising factorial. In 1997, Van Hamme [13] conjectured 13 interesting $p$-adic analogues of Ramanujan-type formulas. For example,

$$
\sum_{k=0}^{(p-1) / 2}(6 k+1) \frac{\left(\frac{1}{2}\right)_{k}^{3}}{k !^{3} 4^{k}} \equiv p(-1)^{(p-1) / 2}\left(\bmod p^{4}\right),
$$

where $p>3$ is a prime. Van Hamme himself gave proofs for three of them. Supercongruences of the form (1.1) are now called Ramanujan-type supercongruences (see [16]). The proof of (1.1) was first provided by Long [9]. See [10] for historical remarks on Van Hamme's 13 supercongruences.

The first author was partially supported by the National Natural Science Foundation of China (grant 11771175). The second author was partially supported by FWF Austrian Science Fund (grant P 32305).

(C) Australian Mathematical Publishing Association Inc. 2021. This is an Open Access article, distributed under the terms of the Creative Commons Attribution licence (http://creativecommons.org/ licenses/by/4.0/), which permits unrestricted re-use, distribution, and reproduction in any medium, provided the original work is properly cited. 
Recently, $q$-supercongruences have been investigated by several authors (see, for example, [3-8, 14, 15]). In particular, in [3], the present authors proved that, for odd integers $d \geqslant 5$,

$$
\sum_{k=0}^{n-1}[2 d k+1] \frac{\left(q ; q^{d}\right)_{k}^{d}}{\left(q^{d} ; q^{d}\right)_{k}^{d}} q^{d(d-3) k / 2} \equiv \begin{cases}0\left(\bmod \Phi_{n}(q)^{2}\right) & \text { if } n \equiv-1(\bmod d), \\ 0\left(\bmod \Phi_{n}(q)^{3}\right) & \text { if } n \equiv-1 / 2(\bmod d) .\end{cases}
$$

Here, we adopt the standard $q$-notation: $[n]=1+q+\cdots+q^{n-1}$ is the $q$-integer; $(a ; q)_{n}=(1-a)(1-a q) \cdots\left(1-a q^{n-1}\right)$ is the $q$-shifted factorial, with the abbreviated notation $\left(a_{1}, a_{2}, \ldots, a_{m} ; q\right)_{n}=\left(a_{1} ; q\right)_{n}\left(a_{2} ; q\right)_{n} \cdots\left(a_{m} ; q\right)_{n}$, and $\Phi_{n}(q)$ stands for the $n$th cyclotomic polynomial in $q$, which may be defined as

$$
\Phi_{n}(q)=\prod_{\substack{1 \leqslant k \leqslant n \\ \operatorname{gcd}(k, n)=1}}\left(q-\zeta^{k}\right),
$$

where $\zeta$ is a primitive $n$th root of unity.

It is worth mentioning that the $q$-congruence (1.2) is not true for $d=3$. In [3], the present authors also gave the following companion of (1.2): for odd integers $d \geqslant 3$ and integers $n>1$,

$$
\sum_{k=0}^{n-1}[2 d k-1] \frac{\left(q^{-1} ; q^{d}\right)_{k}^{d}}{\left(q^{d} ; q^{d}\right)_{k}^{d}} q^{d(d-1) k / 2} \equiv \begin{cases}0\left(\bmod \Phi_{n}(q)^{2}\right) & \text { if } n \equiv 1(\bmod d), \\ 0\left(\bmod \Phi_{n}(q)^{3}\right) & \text { if } n \equiv 1 / 2(\bmod d) .\end{cases}
$$

In this paper, we prove the following $q$-supercongruence, which is a generalisation of the respective second cases of (1.2) and (1.3).

THEOREM 1.1. Let $d$ and $r$ be odd integers satisfying $d \geqslant 3, r \leqslant d-4$ (in particular, $r$ may be negative) and $\operatorname{gcd}(d, r)=1$. Let $n$ be an integer such that $n \geqslant(d-r) / 2$ and $n \equiv-r / 2(\bmod d)$. Then

$$
\sum_{k=0}^{M}[2 d k+r] \frac{\left(q^{r} ; q^{d}\right)_{k}^{d}}{\left(q^{d} ; q^{d}\right)_{k}^{d}} q^{d(d-r-2) k / 2} \equiv 0\left(\bmod \Phi_{n}(q)^{3}\right),
$$

where $M=(d n-2 n-r) / d$ or $n-1$.

Note that, in [5, Theorem 2], the present authors have already proved that (1.4) is true modulo $\Phi_{n}(q)^{2}$ and, further, conjectured that it is also true modulo $\Phi_{n}(q)^{4}$ for $d \geqslant 5$ (see [5, Conjecture 3]). We believe that the full conjecture will be rather difficult to prove.

We apply the method of creative microscoping, recently introduced in a paper by the first author with Zudilin [6], to prove Theorem 1.1. In our application of this method here, we suitably introduce the parameter $a$ (such that the series satisfies the symmetry $a \leftrightarrow a^{-1}$ ) into the terms of the series and prove that the congruence holds modulo $\Phi_{n}(q)$, modulo $1-a q^{n}$ and modulo $a-q^{n}$. Thus, by the Chinese remainder theorem for coprime polynomials, the congruence holds modulo the product $\Phi_{n}(q)\left(1-a q^{n}\right)\left(a-q^{n}\right)$. By letting $a=1$, the congruence is established modulo $\Phi_{n}(q)^{3}$. 
Our paper is organised as follows. In Section 2, we list some tools that we require in our proof of Theorem 1.1. These consist of a lemma about an elementary $q$-congruence modulo a cyclotomic polynomial $\Phi_{n}(q)$, and a very-well-poised Karlsson-Minton type summation by Gasper of which we need a special case. In Section 3, we first prove Theorem 3.1, which is a parametric generalisation of Theorem 1.1 that involves the insertion of different powers of the parameter $a$, appearing in geometric sequences, in the respective $q$-shifted factorials. Afterwards, we show how Theorem 1.1 follows from Theorem 3.1. We conclude with Section 4, where we elaborate on the merits and limits of the method of creative microscoping employed here in the quest of proving [5, Conjecture 3] (which remains open).

\section{Preliminaries}

We need the following result, which is due to the present authors [4, Lemma 2.1]. In order to make the paper self-contained, we include its short proof here.

LEMMA 2.1. Let $d, m$ and $n$ be positive integers with $m \leqslant n-1$. Let $r$ be an integer satisfying $d m \equiv-r(\bmod n)$. Then, for $0 \leqslant k \leqslant m$ and any indeterminate $a$,

$$
\frac{\left(a q^{r} ; q^{d}\right)_{m-k}}{\left(q^{d} / a ; q^{d}\right)_{m-k}} \equiv(-a)^{m-2 k} \frac{\left(a q^{r} ; q^{d}\right)_{k}}{\left(q^{d} / a ; q^{d}\right)_{k}} q^{m(d m-d+2 r) / 2+(d-r) k}\left(\bmod \Phi_{n}(q)\right) .
$$

If $\operatorname{gcd}(d, n)=1$, then the above $q$-congruence also holds for $a=1$.

PROOF. We first assume that $a$ is an indeterminate. Since $q^{d m+r} \equiv q^{n} \equiv 1\left(\bmod \Phi_{n}(q)\right)$,

$$
\begin{aligned}
\frac{\left(a q^{r} ; q^{d}\right)_{m}}{\left(q^{d} / a ; q^{d}\right)_{m}} & =\frac{\left(1-a q^{r}\right)\left(1-a q^{d+r}\right) \cdots\left(1-a q^{d m-d+r}\right)}{\left(1-q^{d} / a\right)\left(1-q^{2 d} / a\right) \cdots\left(1-q^{d m} / a\right)} \\
& \equiv \frac{\left(1-a q^{r}\right)\left(1-a q^{d+r}\right) \cdots\left(1-a q^{d m-d+r}\right)}{\left(1-q^{d-d m-r} / a\right)\left(1-q^{2 d-d m-r} / a\right) \cdots\left(1-q^{-r} / a\right)} \\
& =(-a)^{m} q^{m(d m-d+2 r) / 2}\left(\bmod \Phi_{n}(q)\right) .
\end{aligned}
$$

Moreover, modulo $\Phi_{n}(q)$,

$$
\begin{aligned}
\frac{\left(a q^{r} ; q^{d}\right)_{m-k}}{\left(q^{d} / a ; q^{d}\right)_{m-k}} & =\frac{\left(a q^{r} ; q^{d}\right)_{m}}{\left(q^{d} / a ; q^{d}\right)_{m}} \frac{\left(1-q^{d m-d k+d} / a\right)\left(1-q^{d m-d k+2 d} / a\right) \cdots\left(1-q^{d m} / a\right)}{\left(1-a q^{d m-d k+r}\right)\left(1-a q^{d m-d k+d+r}\right) \cdots\left(1-a q^{d m-d+r}\right)} \\
& \equiv \frac{\left(a q^{r} ; q^{d}\right)_{m}}{\left(q^{d} / a ; q^{d}\right)_{m}} \frac{\left(1-q^{d-d k-r} / a\right)\left(1-q^{2 d-d k-r} / a\right) \cdots\left(1-q^{-r} / a\right)}{\left(1-a q^{-d k}\right)\left(1-a q^{d-d k}\right) \cdots\left(1-a q^{-d}\right)} \\
& =\frac{\left(a q^{r} ; q^{d}\right)_{m}}{\left(q^{d} / a ; q^{d}\right)_{m}} \frac{\left(a q^{r} ; q^{d}\right)_{k}}{\left(q^{d} / a ; q^{d}\right)_{k}} a^{-2 k} q^{(d-r) k} .
\end{aligned}
$$

Substituting (2.1) into this $q$-congruence, we obtain the $q$-congruence in the lemma.

We now assume that $\operatorname{gcd}(d, n)=1$ and $a=1$. Then the desired result follows from the same argument. 
We will further utilise a very-well-poised Karlsson-Minton type summation due to Gasper [1, Equation (5.13)] (see also [2, Example 2.33(i)]): that is,

$$
\begin{aligned}
& \sum_{k=0}^{\infty} \frac{\left(a, q \sqrt{a},-q \sqrt{a}, b, a / b, d, e_{1}, a q^{n_{1}+1} / e_{1}, \ldots, e_{m}, a q^{n_{m}+1} / e_{m} ; q\right)_{k}}{\left(q, \sqrt{a},-\sqrt{a}, a q / b, b q, a q / d, a q / e_{1}, e_{1} q^{-n_{1}}, \ldots, a q / e_{m}, e_{m} q^{-n_{m}} ; q\right)_{k}}\left(\frac{q^{1-v}}{d}\right)^{k} \\
& \quad=\frac{(q, a q, a q / b d, b q / d ; q)_{\infty}}{(b q, a q / b, a q / d, q / d ; q)_{\infty}} \prod_{j=1}^{m} \frac{\left(a q / b e_{j}, b q / e_{j} ; q\right)_{n_{j}}}{\left(a q / e_{j}, q / e_{j} ; q\right)_{n_{j}}}
\end{aligned}
$$

where $n_{1}, \ldots, n_{m}$ are nonnegative integers, $v=n_{1}+\cdots+n_{m}$ and the convergence condition $\left|q^{1-v} / d\right|<1$ is needed when the series does not terminate. We point out that an elliptic extension of the terminating $d=q^{-v}$ case of (2.2) was given by Rosengren and the second author in [12, Equation (1.7)].

In particular, we notice that the right-hand side of (2.2) vanishes for $d=b q$. Further, taking $b=q^{-N}$, we get the summation formula

$$
\sum_{k=0}^{N} \frac{\left(a, q \sqrt{a},-q \sqrt{a}, e_{1}, a q^{n_{1}+1} / e_{1}, \ldots, e_{m}, a q^{n_{m}+1} / e_{m}, q^{-N} ; q\right)_{k}}{\left(q, \sqrt{a},-\sqrt{a}, a q / e_{1}, e_{1} q^{-n_{1}}, \ldots, a q / e_{m}, e_{m} q^{-n_{m}}, a q^{N+1} ; q\right)_{k}} q^{(N-v) k}=0
$$

provided that $N>v=n_{1}+\cdots+n_{m}$.

\section{A parametric generalisation and proof of Theorem 1.1}

We now give a parametric generalisation of Theorem 1.1 .

THEOREM 3.1. Let $d$ and $r$ be odd integers satisfying $d \geqslant 3, r \leqslant d-4$ (in particular, $r$ may be negative) and $\operatorname{gcd}(d, r)=1$. Let $n$ be an integer such that $n \geqslant(d-r) / 2$ and $n \equiv-r / 2(\bmod d)$. Then, modulo $\Phi_{n}(q)\left(1-a q^{n}\right)\left(a-q^{n}\right)$,

$$
\begin{aligned}
& \sum_{k=0}^{M}[2 d k+r] \frac{\left(a^{d-2} q^{r}, a^{d-4} q^{r}, \ldots, a q^{r} ; q^{d}\right)_{k}}{\left(a^{d-2} q^{d}, a^{d-4} q^{d}, \ldots, a q^{d} ; q^{d}\right)_{k}} \\
& \quad \times \frac{\left(a^{2-d} q^{r}, a^{4-d} q^{r}, \ldots, a^{-1} q^{r} ; q^{d}\right)_{k}\left(q^{r} ; q^{d}\right)_{k}}{\left(a^{2-d} q^{d}, a^{4-d} q^{d}, \ldots, a^{-1} q^{d} ; q^{d}\right)_{k}\left(q^{d} ; q^{d}\right)_{k}} q^{d(d-r-2) k / 2} \equiv 0,
\end{aligned}
$$

where $(d n-2 n-r) / d \leqslant M \leqslant n-1$.

PROOF. It is easy to see that $\operatorname{gcd}(d, n)=1$ and, thereby, none of the numbers $d, 2 d, \ldots,(n-1) d$ are multiples of $n$. This means that the denominators of the left-hand side of (3.1) contain neither the factor $1-a q^{n}$ nor $1-a^{-1} q^{n}$. Thus, for 
$a=q^{-n}$ or $a=q^{n}$, the left-hand side of (3.1) can be written as

$$
\begin{aligned}
\sum_{k=0}^{(d n-2 n-r) / d} & {[2 d k+r] \frac{\left(q^{r-(d-2) n}, q^{r-(d-4) n}, \ldots, q^{r-n} ; q^{d}\right)_{k}}{\left(q^{d-(d-2) n}, q^{d-(d-4) n}, \ldots, q^{d-n} ; q^{d}\right)_{k}} } \\
\times & \frac{\left(q^{(d-2) n+r}, q^{(d-4) n+r}, \ldots, q^{n+r} ; q^{d}\right)_{k}\left(q^{r} ; q^{d}\right)_{k}}{\left(q^{(d-2) n+d}, q^{(d-4) n+d}, \ldots, q^{n+d} ; q^{d}\right)_{k}\left(q^{d} ; q^{d}\right)_{k}} q^{d(d-r-2) k / 2},
\end{aligned}
$$

where we have used $\left(q^{r-(d-2) n} ; q^{d}\right)_{k}=0$ for $k>(d n-2 n-r) / d$. Specialising the parameters in (2.3) by $N=(d n-2 n-r) / d, a=q^{r}, q \mapsto q^{d}, m=(d-1) / 2, e_{i}=$ $q^{r-(d-2 i-2) n}(1 \leqslant i \leqslant m-1), e_{m}=q^{(d+r) / 2}, n_{1}=\cdots=n_{m-1}=(2 n+r-d) / d$ and $n_{m}=$ $(2 n+r-d) /(2 d)$ and noticing $N-\left(n_{1}+\cdots+n_{m}\right)=(d-r-2) / 2>0$, we see that (3.2) is equal to 0 . This proves that (3.1) holds modulo $\left(1-a q^{n}\right)\left(a-q^{n}\right)$.

For $M=(d n-2 n-r) / d$, by Lemma 2.1 , we can easily check that

$$
\begin{aligned}
{[2 d(} & M-k)+r] \frac{\left(a^{d-2} q^{r}, a^{d-4} q^{r}, \ldots, a q^{r} ; q^{d}\right)_{M-k}}{\left(a^{d-2} q^{d}, a^{d-4} q^{d}, \ldots, a q^{d} ; q^{d}\right)_{M-k}} \\
& \times \frac{\left(a^{2-d} q^{r}, a^{4-d} q^{r}, \ldots, a^{-1} q^{r} ; q^{d}\right)_{M-k}\left(q^{r} ; q^{d}\right)_{M-k}}{\left(a^{2-d} q^{d}, a^{4-d} q^{d}, \ldots, a^{-1} q^{d} ; q^{d}\right)_{M-k}\left(q^{d} ; q^{d}\right)_{M-k}} q^{d(d-r-2)(M-k) / 2} \\
\equiv & -[2 d k+r] \frac{\left(a^{d-2} q^{r}, a^{d-4} q^{r}, \ldots, a q^{r} ; q^{d}\right)_{k}}{\left(a^{d-2} q^{d}, a^{d-4} q^{d}, \ldots, a q^{d} ; q^{d}\right)_{k}} \\
& \times \frac{\left(a^{2-d} q^{r}, a^{4-d} q^{r}, \ldots, a^{-1} q^{r} ; q^{d}\right)_{k}\left(q^{r} ; q^{d}\right)_{k}}{\left(a^{2-d} q^{d}, a^{4-d} q^{d}, \ldots, a^{-1} q^{d} ; q^{d}\right)_{k}\left(q^{d} ; q^{d}\right)_{k}} q^{d(d-r-2) k / 2}\left(\bmod \Phi_{n}(q)\right) .
\end{aligned}
$$

It now becomes evident that the $k$ th and $(M-k)$ th summands on the left-hand side of (3.1) cancel each other modulo $\Phi_{n}(q)$. Therefore, the left-hand side of (3.1) is congruent to 0 modulo $\Phi_{n}(q)$ for $M=(d n-2 n-r) / d$. Furthermore, for any $k$ in the range $(d n-2 n-r) / d<k \leqslant n-1$, we have $\left(q^{r} ; q^{d}\right)_{k} /\left(q^{d} ; q^{d}\right)_{k} \equiv 0\left(\bmod \Phi_{n}(q)\right)$. Hence, the $q$-congruence (3.1) also holds modulo $\Phi_{n}(q)$ for $(d n-2 n-r) / d<M \leqslant$ $n-1$.

Proof of TheOrem 1.1. Since $\operatorname{gcd}(n, d)=1$ and $0 \leqslant k \leqslant n-1$, the factors related to $a$ in the denominators of the left-hand side of (3.1) are relatively prime to $\Phi_{n}(q)$ when $a=1$. On the other hand, the polynomial $\left(1-a q^{n}\right)\left(a-q^{n}\right)$ has the factor $\Phi_{n}(q)^{2}$ when $a=1$. Thus, letting $a=1$ in (3.1), we see that (1.4) holds modulo $\Phi_{n}(q)^{3}$.

\section{Concluding remarks}

We have inserted different powers of the parameter $a$, appearing in geometric sequences, in the respective $q$-shifted factorials on the left-hand side of (1.4), in order to establish the desired generalised congruence modulo $\left(1-a q^{n}\right)\left(a-q^{n}\right)$. The proof of Theorem 1.1 is similar to the proofs in [3] but is quite different from those in [6], where the parameter $a$ is inserted in a more standard way (without higher powers of $a$ ). 
While the method of creative microscoping enabled us to strengthen [5, Theorem 2] to the congruence modulo $\Phi_{n}(q)^{3}$ in Theorem 1.1, we believe that it is rather unlikely that the validity of (1.4) modulo $\Phi_{n}(q)^{4}$ for $d \geqslant 5$ [5, Conjecture 3] can be proved by the method of creative microscoping, since the parametric generalisation in (3.1) does not hold modulo $\Phi_{n}(q)^{2}\left(1-a q^{n}\right)\left(a-q^{n}\right)$, in general. For this reason, the proof of (1.4) modulo $\Phi_{n}(q)^{2}$ given in [5] still has its virtue. Recall that the present authors, in [5], wrote the left-hand side of (1.4) as a product of two rational functions $X$ and $Y$, and showed that $X$ is congruent to 0 modulo $\Phi_{n}(q)^{2}$. Hence, to prove [5, Conjecture 3], it remains to prove that $Y$ is also congruent to 0 modulo $\Phi_{n}(q)^{2}$. We hope that an interested reader can shed light on this problem and settle the conjecture.

\section{References}

[1] G. Gasper, 'Elementary derivations of summation and transformation formulas for $q$-series', in: Special Functions, q-Series and Related Topics (eds. M. E. H. Ismail, D. R. Masson and M. Rahman), Fields Institute Communications, 14 (Amer. Math. Soc., Providence, RI, 1997), 55-70.

[2] G. Gasper and M. Rahman, Basic Hypergeometric Series, 2nd edn, Encyclopedia of Mathematics and its Applications, 96 (Cambridge University Press, Cambridge, 2004).

[3] V. J. W. Guo and M. J. Schlosser, 'Some new $q$-congruences for truncated basic hypergeometric series', Symmetry 11(2) (2019), Article No. 268, 12 pages.

[4] V. J. W. Guo and M. J. Schlosser, 'A new family of $q$-supercongruences modulo the fourth power of a cyclotomic polynomial', Results Math. 75 (2020), Article No. 155, 13 pages.

[5] V. J. W. Guo and M. J. Schlosser, 'Some $q$-supercongruences modulo the square and cube of a cyclotomic polynomial', Rev. R. Acad. Cienc. Exactas Fís. Nat., Ser. A Mat. 115 (2021), Article No. 132,9 pages.

[6] V. J. W. Guo and W. Zudilin, 'A $q$-microscope for supercongruences', Adv. Math. 346 (2019), 329-358.

[7] L. Li, 'Some $q$-supercongruences for truncated forms of squares of basic hypergeometric series', J. Difference Equ. Appl. 27 (2021), 16-25.

[8] J.-C. Liu and F. Petrov, 'Congruences on sums of $q$-binomial coefficients', Adv. Appl. Math. 116 (2020), Article No. 102003, 11 pages.

[9] L. Long, 'Hypergeometric evaluation identities and supercongruences', Pacific J. Math. 249 (2011), 405-418.

[10] R. Osburn and W. Zudilin, 'On the (K.2) supercongruence of Van Hamme', J. Math. Anal. Appl. 433 (2016), 706-711.

[11] S. Ramanujan, 'Modular equations and approximations to $\pi$ ', Quart. J. Math. Oxford Ser. (2) 45 (1914), 350-372.

[12] H. Rosengren and M. J. Schlosser, 'On Warnaar's elliptic matrix inversion and Karlsson-Minton-type elliptic hypergeometric series', J. Comput. Appl. Math. 178 (2005), 377-391.

[13] L. Van Hamme, 'Some conjectures concerning partial sums of generalized hypergeometric series', in: p-adic Functional Analysis (Nijmegen, 1996), Lecture Notes in Pure and Applied Mathematics, 192 (Dekker, New York, 1997), 223-236.

[14] X. Wang and M. Yue, 'A q-analogue of a Dwork-type supercongruence', Bull. Aust. Math. Soc. 103 (2021), 303-310.

[15] C. Wei, 'Some $q$-supercongruences modulo the fourth power of a cyclotomic polynomial', J. Combin. Theory, Ser. A 182 (2021), Article No. 105469, 15 pages.

[16] W. Zudilin, 'Ramanujan-type supercongruences', J. Number Theory 129(8) (2009), 1848-1857. 
VICTOR J. W. GUO, School of Mathematics and Statistics,

Huaiyin Normal University, Huai' an 223300, Jiangsu, People's Republic of China e-mail:jwguo@hytc.edu.cn

MICHAEL J. SCHLOSSER, Fakultät für Mathematik,

Universität Wien, Oskar-Morgenstern-Platz 1, A-1090 Vienna, Austria

e-mail: michael.schlosser@univie.ac.at 